\title{
Impaired glomerular filtration rate, high grade albuminuria and associated factors among adult patients admitted to tertiary Hospital in Ethiopia
}

\author{
Tamiru Adugna ${ }^{1}$, Hailu Merga $2^{2 *}$ and Esayas Kebede Gudina ${ }^{3}$
}

\begin{abstract}
Background: Chronic Kidney Disease (CKD), the worldwide Public Health problem, is also one of the rising noncommunicable diseases in low and middle-income countries. Its early detection and treatment using readily available, inexpensive therapies can slow or prevent progression to end-stage renal disease. Hence, this study was aimed at assessing impaired estimated glomerular filtration rate (eGFR), high grade albuminuria, and associated factors among adult patients admitted to Jimma University Medical Center in South west Ethiopia.

Methods: Hospital based cross sectional study was conducted from November 1, 2016 to April 30, 2017. Consecutive sampling method was used to select study participants. Logistic regression analysis was conducted to generate factors associated with impaired estimated GFR and albuminuria. A P-value of $<0.05$ was considered statistically significant.

Results: The study involved 422 patients admitted to Jimma University Medical Center who had at least one test result for urinalysis and serum creatinine level during the study period. Fifty two (12.3\%) of the study subjects had high grade albuminuria, 19.2, 19.4, and 32.7\% had impaired estimated glomerular filtration rate according to Modification of Diet in Renal Disease (MDRD-4), Chronic Kidney Disease Epidemiology (CKD-EPI), and Cockcroft-Gault (CG) equations respectively. Old age (AOR $=2.4 ; 95 \% \mathrm{Cl}: 1.4-4.01)$, male sex ( $\mathrm{AOR}=2.1 ; 95 \% \mathrm{Cl}: 1.16-3.7$ ), and hypertension ( $\mathrm{AOR}=2.23$; 95\% Cl:1.24-4.01) were independently associated with impaired eGFR using one of the two equations while diabetes mellitus (AOR $=2.8 ; 95 \% \mathrm{Cl}: 1.33-5.82)$ and $\mathrm{BP}$ measurement above optimal ( $\mathrm{AOR}=4.7 ; 95 \% \mathrm{Cl}: 1.9-11.53)$ were associated with high grade albuminuria.

Conclusions: High grade albuminuria and impaired eGFR were found in significant proportion of adults admitted to the hospital for various medical conditions. Old age, hypertension, diabetes mellitus and male gender were independently associated with these alterations. These findings necessitate routine urinalysis and estimation of GFR for all hospitalized adults with known CKD risk factors.
\end{abstract}

Keywords: Albuminuria, Chronic kidney disease, Creatinine, Glomerular filtration rate, Jimma University medical center

\footnotetext{
* Correspondence: hailu.merga2014@gmail.com

${ }^{2}$ Department of Epidemiology, Institute of Health, Jimma University, Jimma,

Ethiopia

Full list of author information is available at the end of the article
}

(c) The Author(s). 2018 Open Access This article is distributed under the terms of the Creative Commons Attribution 4.0 International License (http://creativecommons.org/licenses/by/4.0/), which permits unrestricted use, distribution, and reproduction in any medium, provided you give appropriate credit to the original author(s) and the source, provide a link to the Creative Commons license, and indicate if changes were made. The Creative Commons Public Domain Dedication waiver (http://creativecommons.org/publicdomain/zero/1.0/) applies to the data made available in this article, unless otherwise stated. 


\section{Background}

Chronic Kidney Disease (CKD), a worldwide Public Health problem, is a gradual loss in kidney function. It is characterized by reduction in Glomerular Filtration Rate (GFR), increased albumin excretion, or both [1]. It is associated with adverse outcome of kidney failure, cardiovascular disease and premature death [2-6]. The global increase of traditional risk factors are the driven factors for the global increase in magnitude of the CKD [7].

Glomerular Filtration Rate (GFR) is a measure of how well kidneys are cleaning blood-taking out extra water and waste. Specifically, it estimates how much blood passes through the glomeruli each minute [8]. It is central to diagnosis and management of Chronic Kidney Disease and accepted as the best overall measure of kidney function. As the clearance of endogenous toxins is affected by various factors, estimated Glomerular Filtration Rate (eGFR) calculated based on serum creatinine level adjusted for patient's age, gender and race is often used as surrogate measures of Glomerular Filtration Rate (eGFR) [9].

Besides eGFR, albuminuria which is the abnormal level of protein in the urine has an important prognostic value and should be included in the evaluation of patients with CKD [10, 11]. Albuminuria is the marker of renal injury which has link with kidney disease progression, increased atherosclerosis, and left ventricular abnormalities indirectly contributing to cardiovascular diseases/disorders and death [4-6].

The preventive strategies of CKD involve identifying those at risk of developing CKD; awareness creation for the peoples on how to prevent renal disease; raising the awareness of the general public, policy makers, and health care workers; modifying the lifestyle of susceptible individuals; detecting early stage of CKD; and hindering the progress of the disease [12].

Even though there are evidences suggesting high burden of CKD in Africa [13], there is paucity of data in sub-Saharan Africa in general and Ethiopia in particular. Moreover, the existing data are hampered by poor quality that limits inferences. This calls for more information and validated measures of kidney function especially in the context of the growing burden of non-communicable diseases in the continent [12].

In Ethiopia, the burden of end-stage renal disease is widely felt by both the public and healthcare providers. However, study about CKD is almost non-existent and the real burden of the disease remains unknown. A small scale facility based cross sectional study conducted in southcentral Ethiopia revealed that at least $18 \%$ of patients with diabetes on follow up have CKD; most of them have never been diagnosed [14].

Early detection and treatment of CKD slow or prevent progression to end stage renal failure in selected groups of patients. Interventions targeting $\mathrm{CKD}$, particularly to reduce urine protein excretion (ACEI and/or ARB) significantly reduced ESRD risk in patients with High Grade proteinuria $[2,3,15,16]$. As routine serum creatinine test and urine dipstick are readily available in most public hospitals in Ethiopia, they can be used along with clinical data to screen high risk groups for CKD. The aim of this study was thus to determine the magnitude of impaired glomerular filtration rate, high grade albuminuria, and associated factors among adult patients admitted to Jimma University Medical Center in southwest Ethiopia. Additionally, the concordance among different serum creatinine based GFR estimating equations was assessed.

\section{Methods}

\section{Study setting}

The study was conducted at Jimma University Medical Center (JUMC) between November 1, 2016 and April 30, 2017. JUMC is one of the oldest public hospitals in the Ethiopia located $352 \mathrm{~km}$ southwest of Addis Ababa. Currently, it is the only teaching and referral hospital in the southwestern part of the country, providing services for about 15 million peoples. Approximately 17,000 inpatients, 218,000 outpatient visits, 14,000 emergency cases and 6000 deliveries in a year coming to the hospital.

\section{Study design and data collection}

Institutional cross-sectional study was conducted to recruit adult patients admitted to Jimma University Medical Center during the study period. However, only adults with at least one test result for Renal Function Test (RFT) and urinalysis were included in this study. Patients with possibility of functional proteinuria, and those with established pre-eclampsia/eclampsia were excluded from study.

The sample size was calculated using a formula for estimation of single population proportion taking prevalence of impaired eGFR and albuminuria to be $50 \%$, margin of error of 5\%, and using 95\% confidence level. Finally, considering the expected patient loss from the study as $10 \%$, the final sample size was calculated to be 422. Consecutive sampling method was used to recruit those study participants.

Data were collected from patients and their record using structured questionnaire which was subdivided into four parts; socio-demographic characteristics, medical history, physical examination and laboratory findings, was developed in English with modification from Screening and Early Evaluation of Kidney Disease (SEEK) study [17]. All laboratory tests were done following the standard procedures recommended by the manufacturer. Serum creatinine was determined using fully automated machine (Horiba Ltd., Japan) and ABX Pentra Creatinine $120 \mathrm{CP}$ reagent using Jaffe's reaction by laboratory technicians. Quality control test was done for all tests and results were 
used for analysis only for those who passed the test. Moreover, all laboratory tests at the hospital are validated continuously by external quality assurance done by Ethiopian Public Health Institute. Data was collected by trained nurses and medical interns with supervision by internal medicine resident from Jimma University Medical Center. During preparatory stage, the questionnaires was carefully designed and pre-tested on $5 \%$ of study population to minimize errors. Based on the result of pretest, revision was made on few contents and concepts of the questionnaire. Data collectors and supervisors were trained for two days prior to data collection period. The collected data was checked for consistency and completeness immediately at the end of the interview.

\section{Measurements}

Acute kidney injury was defined in the study as an increase in serum creatinine $(\mathrm{SCr})$ by $>=0.3 \mathrm{mg} / \mathrm{dl}$ within $48 \mathrm{~h}$; and/or an increase in $\mathrm{SCr}$ to $>=1.5$ times baseline, which is known or presumed to have occurred within the prior 7 days; and/or urine volume $<0.5 \mathrm{ml} / \mathrm{kg} / \mathrm{h}$ for $6 \mathrm{~h}$. Chronic kidney disease was defined as abnormal serum creatinine, eGFR and/or proteinuria which persisted for $>3$ months or eGFR $<60 \mathrm{ml} / \mathrm{min}$ in patients with ultrasonographic finding of shrunken kidney and/or normocytic normochromic anemia. On the other hand, alcohol use problem was defined as drinking alcohol with 'yes' response at least to one of the CAGE assessment question. Smoking status was measured as a person who had used at least 100 cigarettes during their lifetime and who at the time they participated in the study, reported smoking every day or some of the day. Elevated serum creatinine was measured as serum creatinine level $>1.01 \mathrm{mg} / \mathrm{dl}$ for female and $>1.25 \mathrm{mg} / \mathrm{dl}$ for male using NHANES-III cut off point for blacks and end stage renal disease/chronic kidney failure as eGFR< $15 \mathrm{ml} / \mathrm{min}$ using steady state serum creatinine [18]. Impaired estimated glomerular filtration rate (eGFR) was measured in our study as eGFR less than $60 \mathrm{ml} / \mathrm{min}$ using one of the three creatinine based equations; Cockcroft-Gault (CG), Modification of Diet in Renal Disease (MDRD-4) or Chronic Kidney Disease Epidemiology (CKD-EPI). High grade albuminuria was also measured as dipstick proteinuria $>=1+$ excluding functional proteinuria. Khat chewing (Catha edulis) an evergreen plant that is extensively cultivated in the highlands of Ethiopia and surrounding countries, was also measured. A patient was considered a chewer if $\mathrm{s} / \mathrm{he}$ respond 'yes' to the question 'Did you ever chew khat?"

\section{Data processing and analysis}

Collected data was checked for completeness by principal investigator. Serum creatinine based glomerular filtration rate was estimated for all study participants by CG,
MDRD-4 and CKD-EPI equations using QxMD calculator with correction for black race. Urinalysis of all study subjects were revised; after excluding possible causes of functional albuminuria, dipstick proteinuria of $>=+1$ was taken as albuminuria. Finally, the data was entered in to the computer using EpiData software and after verification; it was exported to SPSS (IBM SPSS Statistics for Macintosh, Version 20.0. Armonk, NY) for analysis. Descriptive statistics like percentages, means, medians, standard deviations and ranges were used to describe findings.

A bi-variate analysis was done to sort variables candidate for multiple logistic regression having value less than or equals to 0.25 . Multiple logistic regression analyses were conducted using Backward LR to generate factors associated with the dependent variable. $P$-value $<0.05$ and $95 \%$ confidence interval $(\mathrm{CI})$ and AOR was used in judging the statistical significance of the associations between independent variables and the outcome variable.

Table 1 Socio-demographic characteristics of study participants, Jimma University Medical Center, Ethiopia, 2017

\begin{tabular}{|c|c|c|c|}
\hline $\begin{array}{l}\text { Socio-demographic } \\
\text { characteristics }\end{array}$ & Category & Frequency & Percentage \\
\hline \multirow[t]{4}{*}{ Age } & $<40$ year & 175 & 41.5 \\
\hline & 40-59 year & 122 & 28.9 \\
\hline & $>=60$ year & 125 & 29.6 \\
\hline & Mean \pm SD & $45.37 \pm 18.5$ & \\
\hline \multirow[t]{2}{*}{ Sex } & Male & 215 & 50.9 \\
\hline & Female & 207 & 49.1 \\
\hline \multirow[t]{4}{*}{ Marital status } & Married & 343 & 81.3 \\
\hline & Single & 54 & 12.8 \\
\hline & Widowed & 16 & 3.8 \\
\hline & Divorced & 9 & 2.1 \\
\hline \multirow[t]{2}{*}{ Religion } & Muslim & 293 & 69.4 \\
\hline & Christian & 129 & 30.6 \\
\hline \multirow[t]{7}{*}{ Occupation } & Farmer & 162 & 38.4 \\
\hline & Housewife & 105 & 24.9 \\
\hline & Merchant & 63 & 14.9 \\
\hline & Student & 40 & 9.5 \\
\hline & Employee & 27 & 6.4 \\
\hline & Daily laborer & 12 & 2.8 \\
\hline & Others & 13 & 3.1 \\
\hline \multirow[t]{4}{*}{ Educational status } & Illiterate & 191 & 45.3 \\
\hline & Grade 1-8 & 167 & 39.6 \\
\hline & Grade 9-12 & 31 & 7.3 \\
\hline & College/University & 33 & 7.8 \\
\hline \multirow[t]{2}{*}{ Residence } & Rural & 254 & 60.2 \\
\hline & Urban & 168 & 39.8 \\
\hline
\end{tabular}




\section{Results}

Socio-demographic characteristics of study participants A total of 422 patients were included in the study. About half of the participants (50.9\%) were male. The mean age of the study participants was 45.37 ( $\mathrm{SD}=18.49) ; 29.4 \%$ were 60 years and older. One hundred ninety one (45.3\%) couldn't read and write while those who had completed college and above were less than $10 \%$. About $60 \%$ of them were rural residents and $38.4 \%$ of them were farmers (Table 1).

\section{Clinical and lifestyle characteristics of study participants}

Majority of patients $(\sim 85 \%)$ have only one urine analysis which is both microscopic and dipstick examination of random urine sample. For those having urine analysis more than once, we have used the last one in our study. Majority $(89.8 \%)$ of the study participants were medical patients. Of this $71.3 \%$ had non-communicable disease (NCD) as one of their major diagnosis including $12.8 \%$ patients who were diagnosed with AKI and/or CKD during their hospital stay. The rest of patients (10.2\%) were surgical patients including 10 (2.4\%) who were post-operation state; however, all laboratory data was determined before surgery.

Forty one percent of study participants had BP measurement above optimal at admission; of which $27 \%$ were previously diagnosed to have hypertension. Forty nine (11.6\%) were not checked for hypertension previously of which $4(8.2 \%)$ were diagnosed to have hypertension during admission. Fifty four (12\%) were known diabetic patients. One hundred forty nine (35.3\%) study participants had used Khat; of which about $15.9 \%$ were using it at the time of this study. About one third (33.4\%) of study participants were engaged in regular physical exercise or their work involve significant physical activities. The study revealed that about $6 \%$ (5.9\%) of patients were obese and about $6 \%(6.2 \%)$ of study subjects were HIV patients (Table 2 and Fig. 1).

\section{Pattern of impaired eGFR and albuminuria}

One hundred seventeen (27.7\%) of the study subjects had elevated serum creatinine level; 138 (32.7\%), 81 (19.2\%) and $82(19.4 \%$ had impaired estimated GFR by CG, MDRD-4 and CKD-EPI equations respectively. The study revealed that about $12 \%$ (12.3\%) of the study participants had albuminuria. Moreover, 21.7 and $32.1 \%$ of the study participants with impaired eGFR using CG and MDRD-4 equation respectively had albuminuria (Table 3 ).

\section{Factors associated with impaired eGFR and albuminuria}

Logistic regression analysis was conducted to see the association between dependent and independent variables. Accordingly, on bivariate analysis to look for candidate variables for final model; age $(\mathrm{COR}=3.6$;
Table 2 Life style and clinical characteristics of study participants, Jimma University Medical Center, Ethiopia, 2017

\begin{tabular}{|c|c|c|c|}
\hline $\begin{array}{l}\text { Life style and clinical } \\
\text { characteristics }\end{array}$ & Category & Frequency & Percentage \\
\hline \multirow[t]{3}{*}{ Cigarette smoking } & Never & 400 & 94.8 \\
\hline & Past & 17 & 4.0 \\
\hline & Current & 5 & 1.2 \\
\hline \multirow[t]{5}{*}{ Alcohol drinking } & Never & 378 & 89.6 \\
\hline & Occasionally & 25 & 5.9 \\
\hline & $<3$ times/week & 13 & 3.1 \\
\hline & 3-6 times/week & 4 & 0.9 \\
\hline & Daily & 2 & 0.5 \\
\hline \multirow[t]{2}{*}{ Alcohol use problem } & No & 413 & 97.9 \\
\hline & Yes & 9 & 2.1 \\
\hline \multirow[t]{3}{*}{ Khat chewing } & Never & 273 & 64.7 \\
\hline & Past & 82 & 19.4 \\
\hline & Current & 67 & 15.9 \\
\hline \multirow{2}{*}{$\begin{array}{l}\text { NSAIDs use with } \\
\text { two weeks }\end{array}$} & No & 354 & 83.9 \\
\hline & Yes & 68 & 16.1 \\
\hline \multirow[t]{2}{*}{ History of DM } & No & 368 & 87.2 \\
\hline & Yes & 54 & 12.8 \\
\hline \multirow[t]{3}{*}{ History of hypertension } & No & 259 & 61.4 \\
\hline & Yes & 114 & 27.0 \\
\hline & $\begin{array}{l}\text { Not checked } \\
\text { for previously }\end{array}$ & 49 & 11.6 \\
\hline \multirow[t]{3}{*}{ Physical exercise } & No & 204 & 48.3 \\
\hline & $<150 \mathrm{~min} /$ week & 77 & 18.2 \\
\hline & $>=150 \mathrm{~min} /$ week & 141 & 33.4 \\
\hline \multirow[t]{3}{*}{$\mathrm{BP}$} & $<120 / 80$ & 249 & 59.0 \\
\hline & $120-139 / 80-89$ & 57 & 13.5 \\
\hline & $>=140 / 90$ & 116 & 27.5 \\
\hline \multirow[t]{2}{*}{ BMI } & $<25$ & 397 & 94.1 \\
\hline & $>=25$ & 25 & 5.9 \\
\hline \multirow[t]{2}{*}{ HIV status } & Nonreactive & 396 & 93.8 \\
\hline & Reactive & 26 & 6.2 \\
\hline
\end{tabular}

95\%CI: 2.15-5.9), sex $(\mathrm{COR}=2.1 ; 95 \% \mathrm{CI}: 1.4-3.16)$, residence $\quad(\mathrm{COR}=2.01 ; 95 \% \mathrm{CI}: 1.30-3.1)$, educational status $(\mathrm{COR}=0.46 ; 95 \% \mathrm{CI}: 0.31-0.7)$, occupational status $\quad(\mathrm{COR}=0.55: 95 \% \mathrm{CI}: 0.36-0.83)$, Khat chewing $(\mathrm{COR}=1.7 ; 95 \%$ CI:1.11-2.56), hypertension $(\mathrm{COR}=$ $2.3 ; 95 \% \mathrm{CI}: 1.53-3.5)$, and physical exercise (COR = 1.6;95\%CI:1.02-2.54) were associated with impaired eGFR using CG equations (Table 4).

After multivariable logistic regression analysis using Backward LR, old age $(\mathrm{AOR}=2.376,95 \% \mathrm{CI} 1.38-4.1)$, male gender $(\mathrm{AOR}=1.61,95 \% \mathrm{CI}: 1.03-2.5)$, rural residence $(\mathrm{AOR}=1.9,95 \% \mathrm{CI}: 1.18-3.0)$, and elevated BP 


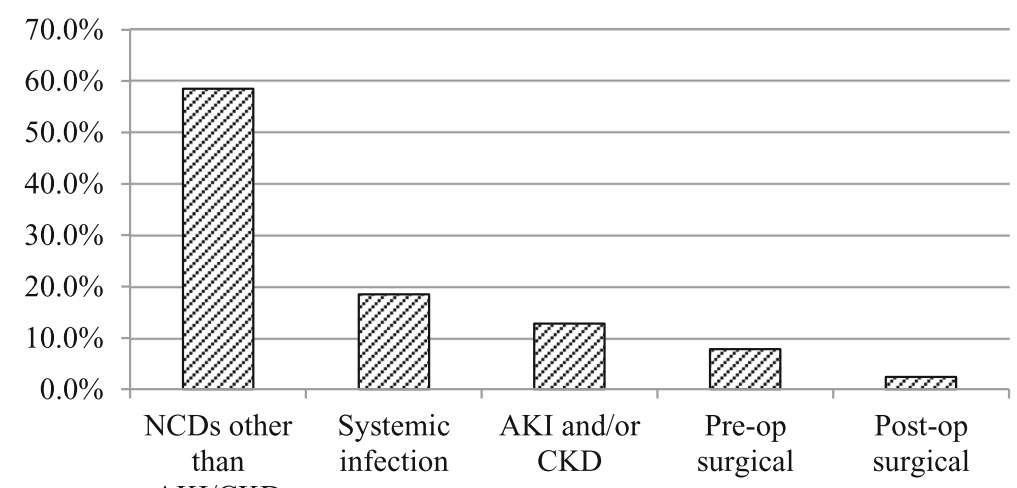

Fig. 1 Admission diagnosis of study participants, Jimma University Medical Center, Ethiopia, 2017

(hypertension) $(\mathrm{AOR}=1.97,95 \% \mathrm{CI}: 1.14-3.4)$ were independently associated with impaired eGFR computed using CG equation (Table 5). On the other hand, age (COR = $1.87 ; 95 \% \mathrm{CI}: 1.04-3.36)$, sex (COR $=2.1 ; 95 \% \mathrm{CI}: 1.26-3.5)$, residence $\quad(\mathrm{COR}=2.53 ; 95 \% \mathrm{CI}: \quad 1.45-4.42)$, occupation $(\mathrm{COR}=0.564 ; 95 \% \mathrm{CI}: 0.35-0.92)$ and hypertension $(\mathrm{COR}=$ 3.6;95\%CI: 2.1-6.1) were associated with impaired eGFR computed by MDRD-4 equation on bi-variate analysis (Table 6). After multivariable logistic regression analysis; male gender $(\mathrm{AOR}=2.1,95 \% \mathrm{CI}: 1.2-3.7)$, rural residence $(\mathrm{AOR}=2.95 ; 95 \% \mathrm{CI}: 1.6-5.6)$, doing moderate exercise $(\mathrm{AOR}=2.3 ; 95 \% \mathrm{CI}: 1.12-4.7)$, and hypertension $(\mathrm{AOR}=$ 2.6; 95\%CI:1.4-4.9) were associated independently with impaired eGFR using MDRD-4 equation (Table 7).

On bi-variate analysis being obesity $(\mathrm{COR}=3.04$; 95\%CI: 1.2-7.7), having history of known hypertension $(\mathrm{COR}=3.2 ; 95 \% \mathrm{CI}: 1.7-5.9)$, and diabetes mellitus $(\mathrm{COR}=3.1 ; 95 \% \mathrm{CI}$ : $1.5-6.1)$ were associated with albuminuria (Table 8). After multi-variate logistic regression analysis, we found that diabetes mellitus $(\mathrm{AOR}=2.8$, 95\%CI:1.3-5.8) and hypertension ( $\mathrm{AOR}=6.303,95 \% \mathrm{CI}$ : 3.1-12.99) were strongly associated with high grade albuminuria (Table 9).

\section{Performance of serum creatinine based equations}

The study showed that the mean $\mathrm{SCr}$ was $1.83 \mathrm{mg} / \mathrm{dl}$ whereas the mean of eGFR by CG, MDRD-4 and CKD-EPI of study subjects were 78.47, 109.4 and 97.34 respectively. The three serum creatinine based equations performance correlate better for low eGFR (Figs. 2-3).

\section{Discussion}

This study revealed that about $28 \%$ (27.7\%) of the patients had elevated serum creatinine level above the cut-off point for blacks. The prevalence of impaired estimated glomerular filtration rate (eGFR $<60 \mathrm{ml} / \mathrm{min}$ ) was $19.2,19.4$ and $32.7 \%$ by MDRD-4, CKD-EPI and CG equations respectively. This result may not reflect the real burden of CKD both in the study setting as well as in the country as it likely included non-steady serum creatinine. A similar study conducted among diabetic patients attending hospital at Southern part of Ethiopia found eGFR $<60 \mathrm{ml} /$ $\mathrm{min} / 1.73 \mathrm{~m} 2$ of 18.2 and $23.8 \%$ according to the MDRD and Cockcroft-Gault (CG) equations [14].

The other systematic and meta-analysis in which hospital based studies were excluded, estimates the prevalence of CKD in Sub Saharan Africa ranged from 2\% in Cote d'Ivoire to 30\% in Zimbabwe with overall prevalence of $13.9 \%$ which is not in line with our finding [12]. The observed difference might be due to differences in study design, population and study setting. The magnitude of impaired eGFR found in this study is comparable with that found by SEEK study [17].

Prevalence of dipstick albuminuria found by our study (12.3\%) roughly goes in line with the study done in tertiary hospital in Lagos, Nigeria which found dipstick proteinuria in $8.3 \%$ of HIV sero-negative and $42.5 \%$ of HIV positive subjects [18].

Old age $(P=.002$, AOR $=2.376)$, and hypertension $(P=.015$, AOR $=1.974)$ were independently associated with two fold increased risk of having impaired eGFR

Table 3 Pattern of high grade albuminuria according to level of eGFR, Jimma University Medical Center, Ethiopia, 2017

\begin{tabular}{|c|c|c|c|c|}
\hline & \multicolumn{2}{|c|}{ eGFR by CG equation } & \multicolumn{2}{|l|}{ eGFR by MDRD-4 equation } \\
\hline & $\begin{array}{l}<60 \mathrm{ml} / \mathrm{min} \\
(n=138)\end{array}$ & $>=60 \mathrm{ml} / \mathrm{min}(n=284)$ & $<60 \mathrm{ml} / \mathrm{min} / 1.73 \mathrm{~m} 2(n=81)$ & $>=60 \mathrm{ml} / \mathrm{min} / 1.73 \mathrm{~m} 2(n=341)$ \\
\hline Albuminuria $(>=+1)$ & $30(21.7 \%)$ & $22(7.7 \%)$ & $26(32.1 \%)$ & $26(7.6 \%)$ \\
\hline
\end{tabular}


Table 4 Bi-variate logistic regression analysis of factors associated with impaired eGFR using CG-equation, Jimma University Medical Center, Ethiopia, 2017

\begin{tabular}{|c|c|c|c|c|c|}
\hline \multirow[t]{2}{*}{ Variables } & & \multicolumn{4}{|c|}{ Impaired eGFR by CG } \\
\hline & & $\begin{array}{l}\text { Yes } \\
n=138\end{array}$ & $\begin{array}{l}\text { No } \\
n=284\end{array}$ & COR $(95 \% \mathrm{Cl})$ & $P$-value \\
\hline \multirow[t]{3}{*}{ Age } & $>=60$ year & $60(43.5 \%)$ & $65(22.9 \%)$ & $3.56(2.146,5.920)$ & .000 \\
\hline & 40-59 year & $42(30.4 \%)$ & $80(28.2 \%)$ & $2.027(1.201,3.421)$ & .008 \\
\hline & $<40$ year & $36(26.1 \%)$ & 139 (48.9\%) & 1 & \\
\hline \multirow[t]{2}{*}{ Sex } & Male & 87 (63.0\%) & $128(45.1 \%)$ & $2.079(1.370,3.156)$ & .001 \\
\hline & Female & $51(37.0 \%)$ & $156(54.9 \%)$ & 1 & \\
\hline \multirow[t]{2}{*}{ Residence } & Rural & $98(71.0 \%)$ & 156 (54.9\%) & $2.01(1.3,3.108)$ & .002 \\
\hline & Urban & $40(29.0 \%)$ & $128(45.1 \%)$ & 1 & \\
\hline \multirow[t]{2}{*}{ Formal education } & Yes & $58(42 \%)$ & $173(60.9 \%)$ & $0.465(.308, .703)$ & .000 \\
\hline & No & $80(58 \%)$ & 111 (39.1\%) & 1 & \\
\hline \multirow[t]{2}{*}{ Occupation } & Non-farmer & $72(52.2 \%)$ & 189 (66.5\%) & $0.548(.362, .830)$ & .005 \\
\hline & Farmer & $66(47.8 \%)$ & $95(33.5 \%)$ & 1 & \\
\hline \multirow[t]{2}{*}{ Cigarette smoking } & Yes & $11(8.0 \%)$ & 11 (3.9\%) & $2.150(.908,5.089)$ & .082 \\
\hline & No & 127 (92.0\%) & $273(96.1 \%)$ & 1 & \\
\hline \multirow[t]{2}{*}{ Khat chewing } & Yes & $60(43.5 \%)$ & $89(31.3 \%)$ & $1.685(1.108,2.564)$ & .015 \\
\hline & No & $78(56.5 \%)$ & 195 (68.7\%) & 1 & \\
\hline \multirow[t]{3}{*}{ Physical exercise } & $>=150 \mathrm{~min} /$ week & 55 (39.9\%) & $86(30.3 \%)$ & $1.610(1.021,2.538)$ & .040 \\
\hline & $<150 \mathrm{~min} /$ week & $25(18.1 \%)$ & $52(18.3 \%)$ & $1.210(.687,2.131)$ & .509 \\
\hline & No & $58(42.0 \%)$ & $146(51.4 \%)$ & 1 & \\
\hline \multirow[t]{2}{*}{ History of known HTN } & Yes & $72(52.2 \%)$ & $91(32.0 \%)$ & $2.31(1.525,3.510)$ & .000 \\
\hline & No & $66(47.8 \%)$ & $193(68.0 \%)$ & 1 & \\
\hline \multirow[t]{3}{*}{$\mathrm{BP}(\mathrm{mmHg})$} & $>=140 / 90$ & $54(39.1 \%)$ & $62(21.8 \%)$ & $2.805(1.758,4.476)$ & .000 \\
\hline & $120-139 / 80-89$ & $25(18.1 \%)$ & $32(11.3 \%)$ & $2.52(1.382,4.581)$ & .003 \\
\hline & $<120 / 80$ & $59(42.8 \%)$ & 190 (66.9\%) & 1 & \\
\hline
\end{tabular}

Table 5 Multi-variable logistic regression analysis of factors associated with impaired eGFR using CG equation, Jimma University Medical Center, Ethiopia, 2017

\begin{tabular}{|c|c|c|c|c|}
\hline & & COR $(95 \% \mathrm{Cl})$ & AOR (95\%Cl) & $P$-value \\
\hline \multirow[t]{3}{*}{ Age } & $>=60$ year & $3.564(2.146,5.920)$ & $2.376(1.378,4.095)$ & 0.002 \\
\hline & 40-59year & $2.027(1.201,3.421)$ & & \\
\hline & $<40$ year & 1 & & \\
\hline \multirow[t]{2}{*}{ Sex } & Male & $2.079(1.370,3.156)$ & $1.609(1.029,2.515)$ & 0.037 \\
\hline & Female & 1 & & \\
\hline \multirow[t]{2}{*}{ Residence } & Rural & $2.01(1.3,3.108)$ & $1.882(1.181,3.000)$ & 0.008 \\
\hline & Urban & 1 & & \\
\hline \multirow[t]{3}{*}{$\mathrm{BP}(\mathrm{mmHg})$} & $>=140 / 90$ & $2.805(1.758,4.476)$ & $1.974(1.142,3.411)$ & 0.015 \\
\hline & 120-139/80-89 & $2.516(1.382,4.581)$ & $2.112(1.114,4.025)$ & 0.022 \\
\hline & $<120 / 80$ & 1 & & \\
\hline
\end{tabular}


Table 6 Bi-variate logistic regression analysis of factors associated with impaired eGFR by MDRD-4 equation, Jimma University Medical Center, Ethiopia, 2017

\begin{tabular}{|c|c|c|c|c|c|}
\hline \multirow[t]{2}{*}{ Variables } & & \multicolumn{4}{|c|}{ Impaired eGFR by MDRD } \\
\hline & & $\begin{array}{l}\text { Yes } \\
\mathrm{n}=81\end{array}$ & $\begin{array}{l}\text { No } \\
\mathrm{n}=341\end{array}$ & $\operatorname{COR}(95 \% \mathrm{Cl})$ & $P$-value \\
\hline \multirow[t]{3}{*}{$\overline{\text { Age }}$} & $>=60$ year & $25(30.9 \%)$ & $100(29.3 \%)$ & $1.433(.783,2.623)$ & 0.244 \\
\hline & 40-59 year & $30(37.0 \%)$ & $92(27.0 \%)$ & $1.869(1.040,3.358)$ & 0.036 \\
\hline & $<40$ year & $26(32.1 \%)$ & 149 (43.7\%) & 1 & \\
\hline \multirow[t]{2}{*}{ Sex } & Male & $53(65.4 \%)$ & $162(47.5 \%)$ & $2.091(1.262,3.465)$ & 0.004 \\
\hline & Female & $28(34.6 \%)$ & $179(52.5 \%)$ & 1 & \\
\hline \multirow[t]{2}{*}{ Residence } & Rural & $62(76.5 \%)$ & $192(56.3 \%)$ & $2.532(1.451,4.419)$ & 0.001 \\
\hline & Urban & $19(23.5 \%)$ & $149(43.7 \%)$ & 1 & \\
\hline \multirow[t]{2}{*}{ Formal education } & Yes & $38(46.9 \%)$ & $193(56.6 \%)$ & $.678(.417,1.102)$ & 0.117 \\
\hline & No & $43(53.1 \%)$ & $148(43.4 \%)$ & 1 & \\
\hline \multirow[t]{2}{*}{ Occupational status } & Farmer & $40(49.4 \%)$ & $121(35.5 \%)$ & 1 & \\
\hline & Others & $41(50.6 \%)$ & $220(64.5 \%)$ & $.564(.346, .919)$ & 0.022 \\
\hline \multirow[t]{2}{*}{ Cigarette smoking } & Yes & $7(.6 \%)$ & $15(4.4 \%)$ & $2.056(.810,5.221)$ & 0.130 \\
\hline & No & $74(91.4 \%)$ & $326(95.6 \%)$ & 1 & \\
\hline \multirow[t]{3}{*}{ Physical exercise } & $>=150 \mathrm{~min}$ & $31(38.3 \%)$ & $110(32.3 \%)$ & $1.515(.875,2.622)$ & 0.138 \\
\hline & $<150 \mathrm{~min} /$ week & $18(22.2 \%)$ & $59(17.3 \%)$ & $1.640(.857,3.137)$ & 0.135 \\
\hline & No & $32(39.5 \%)$ & $172(50.4 \%)$ & 1 & \\
\hline \multirow[t]{2}{*}{ History of known HTN } & Yes & $43(53.1 \%)$ & $113(33.1 \%)$ & $3.254(1.971,5.374)$ & 0.000 \\
\hline & No & $38(47.9 \%)$ & $228(66.9 \%)$ & 1 & \\
\hline \multirow[t]{2}{*}{ NSAIDs use within two weeks } & Yes & $8(9.9 \%)$ & $60(17.6 \%)$ & $.513(.235,1.121)$ & 0.094 \\
\hline & No & $73(90.1 \%)$ & $281(82.4 \%)$ & 1 & \\
\hline \multirow[t]{3}{*}{$\mathrm{BP}(\mathrm{mmHg})$} & $>=140 / 90$ & $39(48.1 \%)$ & $77(22.6 \%)$ & $3.562(2.079,6.103)$ & 0.000 \\
\hline & $120-139 / 80-89$ & $11(13.6 \%)$ & $46(13.5 \%)$ & $1.682(.788,3.588)$ & 0.179 \\
\hline & $<120 / 80$ & $31(38.3 \%)$ & $218(63.9 \%)$ & 1 & \\
\hline
\end{tabular}

Table 7 Multi-variable logistic regression analysis of factors associated with impaired eGFR by MDRD-4 equation, Jimma University Medical center, Ethiopia, 2017

\begin{tabular}{|c|c|c|c|c|}
\hline \multicolumn{2}{|l|}{ Variables } & \multirow{2}{*}{$\frac{\operatorname{COR}(95 \% \mathrm{Cl})}{2.091(1.262,3.465)}$} & \multirow{2}{*}{$\frac{\operatorname{AOR}(95 \% \mathrm{Cl})}{2.084(1.167,3.721)}$} & \multirow{2}{*}{$\begin{array}{l}\begin{array}{l}P \text { - } \\
\text { value }\end{array} \\
0.013\end{array}$} \\
\hline Sex & Male & & & \\
\hline & Female & 1 & 1 & \\
\hline \multirow[t]{2}{*}{ Residence } & Rural & $2.532(1.451,4.419)$ & $2.954(1.556,5.609)$ & 0.001 \\
\hline & Urban & 1 & & \\
\hline \multirow[t]{3}{*}{ Physical exercise } & $>=150 \mathrm{~min}$ & $1.515(.875,2.622)$ & $.902(.473,1.721)$ & 0.754 \\
\hline & $<150 \mathrm{~min} /$ week & $1.640(.857,3.137)$ & $2.290(1.120,4.685)$ & 0.023 \\
\hline & No & 1 & & \\
\hline \multirow[t]{2}{*}{ History of known HTN } & Yes & $3.254(1.971,5.374)$ & $2.233(1.244,4.010)$ & 0.007 \\
\hline & No & 1 & & \\
\hline \multirow[t]{3}{*}{$\mathrm{BP}(\mathrm{mmHg})$} & $>=140 / 90$ & $3.562(2.079,6.103)$ & $2.597(1.378,4.893)$ & 0.003 \\
\hline & 120-139/80-89 & $1.682(.788,3.588)$ & $1.676(0.757,3.706)$ & 0.203 \\
\hline & $<120 / 80$ & 1 & & \\
\hline
\end{tabular}


Table 8 Bi-variate logistic regression analysis of factors associated with high grade albuminuria, JUMC, Ethiopia, 2017

\begin{tabular}{|c|c|c|c|c|c|}
\hline \multirow[t]{2}{*}{ Variables } & & \multicolumn{4}{|c|}{ High grade albuminuria } \\
\hline & & Yes $n=52$ & No $n=370$ & COR $(95 \% \mathrm{Cl})$ & $P$-value \\
\hline \multirow[t]{3}{*}{ Age } & $>=60$ year & $14(26.9 \%)$ & $111(30.0 \%)$ & $.977(.473,2.019)$ & 0.951 \\
\hline & 40-59 year & $18(34.6 \%)$ & $104(28.1 \%)$ & $1.341(.677,2.657)$ & 0.400 \\
\hline & $<40$ year & $20(38.5 \%)$ & 155 (41.9\%) & 1 & \\
\hline \multirow[t]{2}{*}{ Sex } & Male & $32(61.5 \%)$ & $183(49.5 \%)$ & $1.635(.902,2.96)$ & 0.105 \\
\hline & Female & $20(38.5 \%)$ & $187(50.5 \%)$ & 1 & \\
\hline \multirow[t]{2}{*}{ Alcohol drinking } & Yes & $9(17.3 \%)$ & $35(9.5 \%)$ & $2.0(.902,4.451)$ & 0.088 \\
\hline & No & $43(82.7 \%)$ & $335(90.5 \%)$ & 1 & \\
\hline \multirow[t]{2}{*}{$\mathrm{BMI}$} & $>=25$ & $7(13.5 \%)$ & $18(4.9 \%)$ & $3.04(1.204,7.68)$ & 0.019 \\
\hline & $<25$ & $45(86.5 \%)$ & $352(95.1 \%)$ & 1 & \\
\hline \multirow[t]{2}{*}{ History of known HTN } & Yes & $33(63.5 \%)$ & $130(35.1 \%)$ & $3.206(1.754,5.86)$ & 0.000 \\
\hline & No & 19 (36.5\%) & $240(64.9 \%)$ & 1 & \\
\hline \multirow[t]{2}{*}{ History of DM } & Yes & $14(26.9 \%)$ & $40(10.8 \%)$ & $3.039(1.517,6.09)$ & 0.002 \\
\hline & No & $38(73.1 \%)$ & $330(89.2 \%)$ & 1 & \\
\hline \multirow[t]{3}{*}{$\mathrm{BP}(\mathrm{mmHg})$} & $>=140 / 90$ & $29(55.7 \%)$ & $87(23.5 \%)$ & $6.583(3.21,13.47)$ & 0.000 \\
\hline & $120-139 / 80-89$ & $11(21.2 \%)$ & $46(12.4 \%)$ & $4.723(1.96,11.3)$ & 0.001 \\
\hline & $<120 / 80$ & $12(23.1 \%)$ & $237(64.1 \%)$ & 1 & \\
\hline
\end{tabular}

by CG equation. This is consistent with other similar studies $[19,20]$.

Our study has also shown that rural residence $(P=$ $0.001, \mathrm{AOR}=2.954)$ and male gender $(P=0.013, \mathrm{AOR}=$ 2.084) were associated with lower eGFR by both CG and MDRD-4 equations. Most studies show that the risk of CKD increases in urban settings than rural residents [21]. The risk with rural residence in our study may be due to poor living standard and increased risk of infection both of which beget higher risk of glomerulonephritis. However, there are no good quality data that compare differences with residence in Africa [12]. Similarly, the link with male sex is contrary to previous reports which have shown higher eGFR impairment in female [14, 17, 19].

Moderate physical exercise $(P=.023$, AOR $=2.29)$ was associated with 2 folds increased risk of having impaired
eGFR computed by MDRD-4 equation. This finding is inconsistent with studies from North India and Screening and Early Evaluation of Kidney Disease (SEEK) study [17]. This might be due to selection bias.

Individuals who had diabetes mellitus $(P=.006$, $\mathrm{AOR}=2.785$ ) were about three times more likely to have high grade albuminuria. On the other hand, BP measurement above optimal $(P=.001, \mathrm{AOR}=4.757)$ had increased risk of high grade albuminuria by more than four folds [2, 4, 19, 22, 23].

However, cigarette smoking $(P=.082)$, alcohol $(P=.088)$, NSAIDs $(P=.094)$ and HIV/AIDS $(P=.605)$ were not associated with any of the study outcomes likely due to small sample size.

In this study, the prevalence of impaired eGFR using CG-equation is higher than that of impaired eGFR by MDRD-4 and CKD-EPI equations. On the other hand,

Table 9 Multi-variable logistic analysis of factors associated with high grade albuminuria, Jimma University Medical Center, Ethiopia, 2017

\begin{tabular}{|c|c|c|c|c|}
\hline \multirow[t]{2}{*}{ Variables } & & \multicolumn{3}{|l|}{ OR with $95 \% \mathrm{Cl}$} \\
\hline & & $\overline{\operatorname{COR}(95 \% \mathrm{Cl})}$ & AOR $(95 \% \mathrm{Cl})$ & $P$-value \\
\hline \multirow[t]{2}{*}{ History of DM } & Yes & $3.039(1.517,6.091)$ & $2.785(1.332,5.825)$ & 0.006 \\
\hline & No & 1 & & \\
\hline \multirow[t]{3}{*}{$\mathrm{BP}(\mathrm{mmHg})$} & $>=140 / 90$ & $6.583(3.21,13.474)$ & $6.303(3.059,12.987)$ & 0.000 \\
\hline & $120-139 / 80-89$ & $4.723(1.965,11.352)$ & $4.757(1.962,11.533)$ & 0.001 \\
\hline & $<120 / 80$ & 1 & & \\
\hline
\end{tabular}




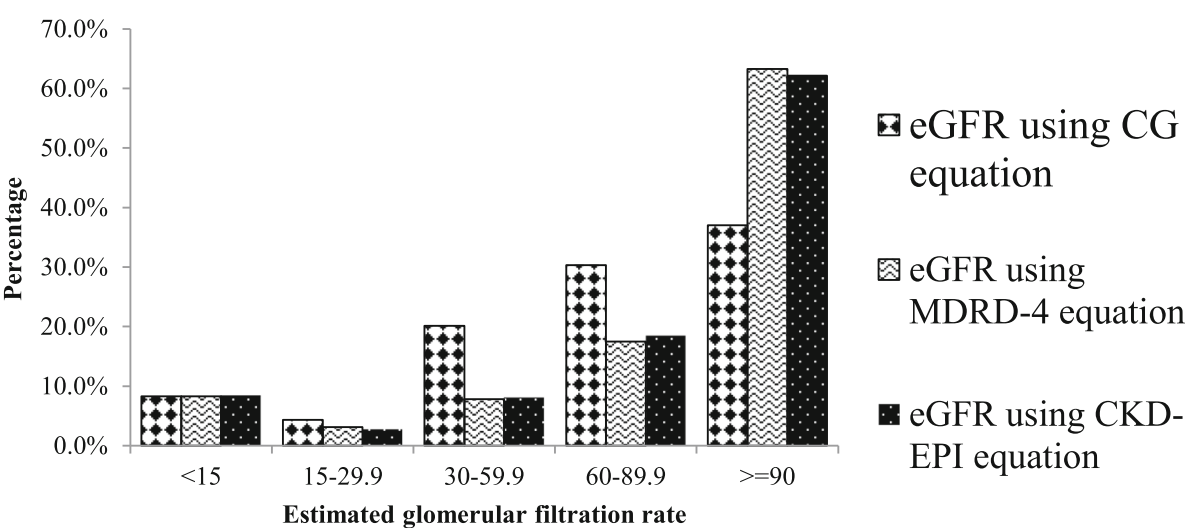

Fig. 2 Estimated GFR using serum creatinine based equations, Jimma University Medical Center, Ethiopia, 2017

MDRD-4 and CKD-EPI equations performed closely. This is roughly in line with the study from South-Africa which found that highest agreement between GFR estimators was between MDRD and CKD-EPI equations [12, 17, 22].

More than $70 \%$ of the study participants had Non-Communicable Diseases (NCDs) as one of their admission diagnoses. Forty one percent (41\%) of patients had BP above optimal (BP $>=120 / 80 \mathrm{mmHg}$ ) of which more than $27 \%$ were hypertensive while at least $12 \%$ of the study participants had diabetes mellitus. On the other hand, we found that more than $19 \%$ of the study participants had eGFR $<60 \mathrm{ml} / \mathrm{min}$ by all three equations while more than $12 \%$ had dipstick proteinuria. These are the reflection of double burden of NCDs on developing nations like Ethiopia where there are limited facilities to care for chronic diseases like ESRD. These all necessitates timely detection and treatment of NCDs in general and CKD in particular and their risk factors.

Even though this study has many strengths, it has few limitations. As the study design used was institution based cross sectional study, it is impossible to infer for the general population. The other limitation is that our serum creatinine based eGFR calculation likely included unsteady serum creatine level. There is also

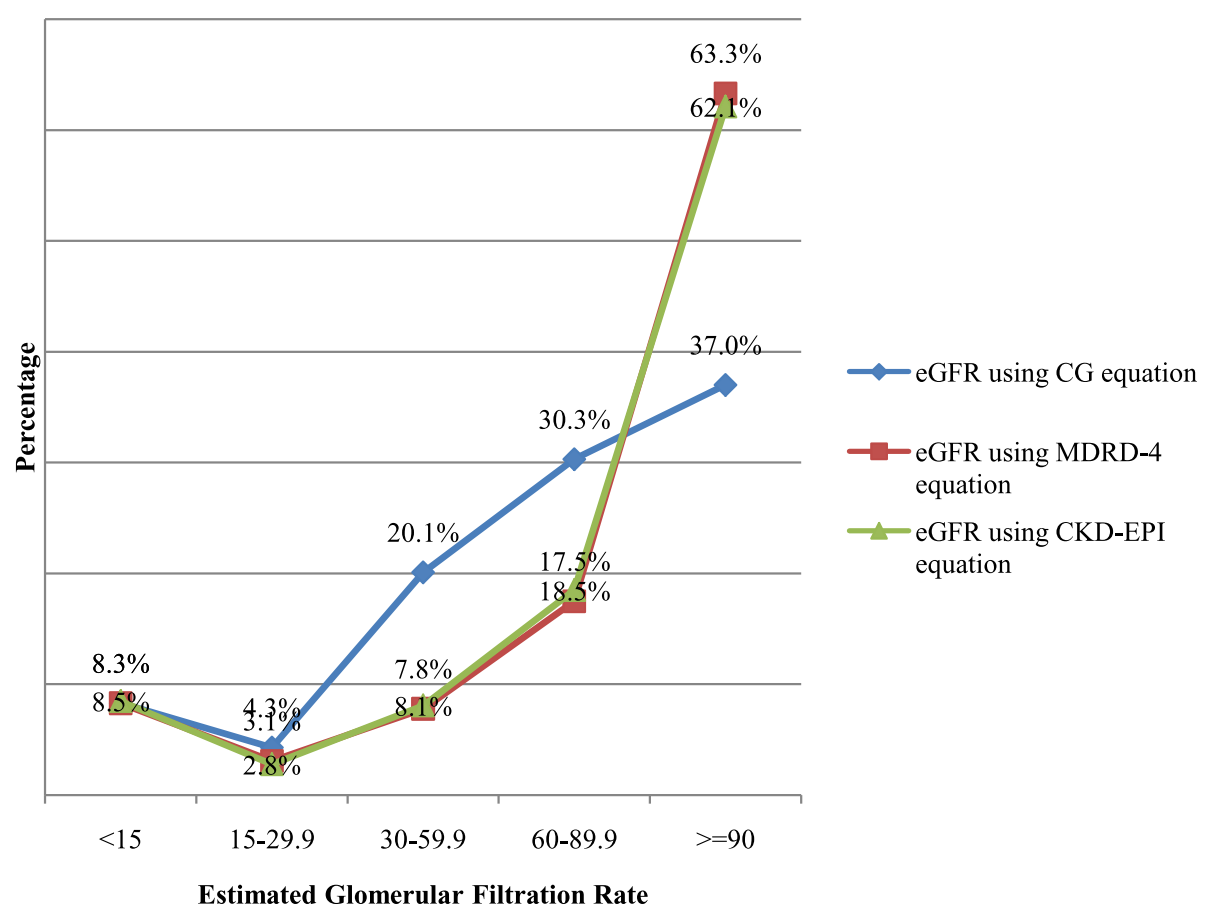

Fig. 3 Comparison of the three serum creatinine based equations, Jimma University Medical Center, Ethiopia, 2017 
a possibility of functional proteinuria like orthostatic proteinuria as we used random urine sample. Notwithstanding these limitations, we believe that our study has very important findings in the study area and areas with similar set up.

\section{Conclusions}

Impaired estimated Glomerular Filtration Rate and High grade albuminuria of all grades was found in significant proportion of study participants which necessitates routine urine analysis and estimation of Glomerular Filtration Rate for patients with CKD risk factors. On the other hand, MDRD-4 and CKD-EPI equations perform comparable in estimating GFR through the range of eGFR while CG equation correlate better with other equations when eGFR below 30 $\mathrm{ml} / \mathrm{min}$. Our study showed that old age, being male sex, and hypertension were independently associated with impaired eGFR using one of the two equations while being diabetic and having BP measurement above optimal were associated with high grade albuminuria. Therefore, Health workers should be vigilant in utilizing available resources to detect risk factors of NCDs including CKD and foster healthy life style of their clients through health education. Estimation of GFR and urine analysis should be routine for patients with traditional risk factors for CKD and urologic diseases.

\section{Abbreviations \\ ACR: Albumin-creatinine ratio; AKI: Acute Kidney Injury; BMI: Body Mass Index; CAGE: Cut down, Annoyed, Guilty, Eye opener; CG: Cockcroft-Gault equation; CKD: Chronic kidney disease; CrCl: Creatinine clearance; CVD: Cardiovascular disease; DKA: Diabetic Keto-acidosis; DM: Diabetic mellitus; eGFR: Estimated glomerular filtration rate; ESRD: End-stage renal disease; FBS: Fasting blood sugar; GFR: Glomerular filtration rate; JUMC: Jimma University Medical Center; KDOQI: Kidney Disease Outcomes Quality Initiative; MDRD: Modified Diet Renal Disease; RFT: Renal function test; SCr: Serum creatinine; WHO: World Health Organization}

\section{Acknowledgments}

We would like to thank Jimma University Institute of Health for financial support for the realization of this finding. We are also grateful for Jimma University Medical Center for the provision of the needed data for our study. Special thanks and appreciation to all those who agreed to participate in this study, mainly respondents, data collectors and supervisors.

\section{Funding}

This research was funded by Jimma University Institute of Health. The organization has no any role in the designing of the study, data collection, analysis, and interpretation of data and in writing the manuscript.

\section{Availability of data and materials}

Data will be available upon request from the corresponding author.

\section{Authors' contributions}

TA involved in conception, designing methods, analysis, interpretation and drafting of the manuscript. EKG and HM Participated in designing, data analysis, interpretation of the findings and write-up of the findings. All authors have reviewed and approved the submission of the manuscript.

\section{Authors' information}

TA: MD, Internist, Assistant professor of Medicine, College of health Sciences, Ambo University.

HM: MPH, Lecturer of Epidemiology, department of Epidemiology, Institute of Health, Jimma University.

EK: MD, DTM \& H, PhD, Associate professor of Medicine, Institute of Health, Jimma University.

\section{Ethics approval and consent to participate}

Ethical clearance was obtained first from Institutional Review Board (IRB) of the Jimma University Institute of Health. Then, appropriate letter of support from the Jimma University Medical Center director was taken to the concerned body prior to data collection. Purpose and significance of the study was explained and written informed consent was obtained from each study participants or their caregivers. Patients' privacy, equity of services and interests of patients was ensured during the study period by informing the data collectors on ethical issues. This study did not involve any potentially harmful intervention to the patient. Confidentiality of the collected data was ensured through anonymity.

\section{Consent for publication}

Not applicable as it does not contain an individual person's data.

Competing interests

The authors declare that they have no competing interests.

\section{Publisher's Note}

Springer Nature remains neutral with regard to jurisdictional claims in published maps and institutional affiliations.

\section{Author details}

${ }^{1}$ Department of Internal medicine, College of Health Sciences, Ambo University, Ambo, Ethiopia. 'Department of Epidemiology, Institute of Health, Jimma University, Jimma, Ethiopia. ${ }^{3}$ Department of Internal medicine, Institute of Health, Jimma University, Jimma, Ethiopia.

Received: 10 August 2018 Accepted: 23 November 2018

Published online: 04 December 2018

\section{References}

1. Jha V, Garcia-Garcia G, Iseki K, Li Z, Naicker S, Plattner B, et al. Chronic kidney disease: global dimension and perspectives. Lancet. 2013;382(9888):260-72.

2. Stevens PE, Levin A. Kidney Disease: Improving global outcomes chronic kidney disease guideline development work group M. evaluation and management of chronic kidney disease: synopsis of the kidney disease: improving global outcomes 2012 clinical practice guideline. Ann Intern Med. 2013;158(11):825-30.

3. Levey AS, Coresh J, Balk E, Kausz AT, Levin A, Steffes MW, et al. National Kidney Foundation practice guidelines for chronic kidney disease: evaluation, classification, and stratification. Ann Intern Med. 2003;139(2):137-47.

4. Matsushita K, Coresh J, Sang Y, Chalmers J, Fox C, Guallar E, et al. Estimated glomerular filtration rate and albuminuria for prediction of cardiovascular outcomes: a collaborative meta-analysis of individual participant data. Lancet Diabetes Endocrinol. 2015;3(7):514-25.

5. Segura J, Campo C, Ruilope LM. Effect of proteinuria and glomerula filtration rate on cardiovascular risk in essential hypertension. Kidney Int Suppl. 2004;66(92):S45-9.

6. Herzog CA, Asinger RW, Berger AK, Charytan DM, Diez J, Hart RG, et al. Cardiovascular disease in chronic kidney disease. A clinical update from kidney disease: improving global outcomes (KDIGO). Kidney Int. 2011;80(6):572-86.

7. Ayodele $\mathrm{OE}$, Alebiosu CO. Burden of chronic kidney disease: an international perspective. Adv Chronic Kidney Dis. 2010;17(3):215-24.

8. Traynor J, Mactier R, Geddes CC, Fox JG. How to measure renal function in clinical practice. BMJ. 2006;333(7571):733-7.

9. Lamb EJ, Brettell EA, Cockwell P, Dalton N, Deeks JJ, Harris K, et al. The eGFR-C study: accuracy of glomerular filtration rate (GFR) estimation using creatinine and cystatin $C$ and albuminuria for monitoring disease progression in patients with stage 3 chronic kidney disease--prospective longitudinal study in a multiethnic population. BMC Nephrol. 2014;15:13.

10. Gansevoort RT, de Jong PE. The case for using albuminuria in staging chronic kidney disease. J Am Soc Nephrol. 2009;20(3):465-8. 
11. Kunimura A, Ishii H, Uetani T, Harada K, Kataoka T, Takeshita M, et al. Prognostic value of albuminuria on cardiovascular outcomes after elective percutaneous coronary intervention. Am J Cardiol. 2016;117(5):714-9.

12. Stanifer JW, Jing B, Tolan S, Helmke N, Mukerjee R, Naicker S, et al. The epidemiology of chronic kidney disease in sub-Saharan Africa: a systematic review and meta-analysis. Lancet Glob Health. 2014;2(3):e174-81.

13. Naicker S. Burden of end-stage renal disease in sub-Saharan Africa. Clin Nephrol. 2010;74(Suppl 1):S13-6.

14. Fiseha T, Kassim M, Yemane T. Chronic kidney disease and underdiagnosis of renal insufficiency among diabetic patients attending a hospital in southern Ethiopia. BMC Nephrol. 2014;15:198.

15. Fink HA, Ishani A, Taylor BC, Greer NL, MacDonald R, Rossini D, et al. Screening for, monitoring, and treatment of chronic kidney disease stages 1 to 3: a systematic review for the U.S. preventive services task force and for an American College of Physicians Clinical Practice Guideline. Ann Intern Med. 2012;156(8):570-81.

16. Couser WG, Remuzzi G, Mendis S, Tonelli M. The contribution of chronic kidney disease to the global burden of major noncommunicable diseases. Kidney Int. 2011;80(12):1258-70.

17. Singh AK, Farag YM, Mittal BV, Subramanian KK, Reddy SR, Acharya VN, et al. Epidemiology and risk factors of chronic kidney disease in India - results from the SEEK (screening and early evaluation of kidney disease) study. BMC Nephrol. 2013;14:114.

18. Lesi O, Bamidele O, Amira C, Okany C, Akanmu A. Prevalence and utility of dipstick proteinuria in predicting renal insufficiency in treatment naive human immunodeficiency virus (HIV) infected Africans. Journal of AIDS and HIV Research. 2014;6(3):53-9.

19. Zhang QL, Rothenbacher D. Prevalence of chronic kidney disease in population-based studies: systematic review. BMC Public Health. 2008;8:117.

20. Simal F, Martin Escudero JC, Bellido J, Arzua D, Mena FJ, Gonzalez Melgosa I, et al. Prevalence of mild to moderate chronic kidney disease in the general population of Spain. Hortega study. Nefrologia. 2004;24(4):329-32.

21. Adjei DN, Stronks K, Adu D, Beune E, Meeks K, Smeeth L, et al. Chronic kidney disease burden among African migrants in three European countries and in urban and rural Ghana: the RODAM cross-sectional study. Nephrol Dial Transplant. 2018;33(10):1812-22.

22. Matsha TE, Yako YY, Rensburg MA, Hassan MS, Kengne AP, Erasmus RT. Chronic kidney diseases in mixed ancestry south African populations: prevalence, determinants and concordance between kidney function estimators. BMC Nephrol. 2013;14:75.

23. Chen F, Yang W, Weng J, Jia W, Ji L, Xiao J, et al. Albuminuria: prevalence, associated risk factors and relationship with cardiovascular disease. J Diabetes Investig. 2014;5(4):464-71

Ready to submit your research? Choose BMC and benefit from:

- fast, convenient online submission

- thorough peer review by experienced researchers in your field

- rapid publication on acceptance

- support for research data, including large and complex data types

- gold Open Access which fosters wider collaboration and increased citations

- maximum visibility for your research: over $100 \mathrm{M}$ website views per year

At $\mathrm{BMC}$, research is always in progress.

Learn more biomedcentral.com/submissions 